\title{
Storm track sensitivity to sea surface temperature resolution in a regional atmosphere model
}

\author{
Tim Woollings · Brian Hoskins • Mike Blackburn • \\ David Hassell · Kevin Hodges
}

Received: 1 December 2008/Accepted: 17 March 2009/Published online: 10 April 2009

(C) Springer-Verlag 2009

\begin{abstract}
A high resolution regional atmosphere model is used to investigate the sensitivity of the North Atlantic storm track to the spatial and temporal resolution of the sea surface temperature (SST) data used as a lower boundary condition. The model is run over an unusually large domain covering all of the North Atlantic and Europe, and is shown to produce a very good simulation of the observed storm track structure. The model is forced at the lateral boundaries with 15-20 years of data from the ERA-40 reanalysis, and at the lower boundary by SST data of differing resolution. The impacts of increasing spatial and temporal resolution are assessed separately, and in both cases increasing the resolution leads to subtle, but significant changes in the storm track. In some, but not all cases these changes act to reduce the small storm track biases
\end{abstract}

T. Woollings $(\bowtie) \cdot$ B. Hoskins $\cdot$ M. Blackburn Department of Meteorology, Walker Institute, University of Reading, Earley Gate, PO Box 243, Reading RG6 6BB, UK e-mail: t.j.woollings@rdg.ac.uk

T. Woollings $\cdot$ M. Blackburn

National Centre for Atmospheric Science, Reading, UK

B. Hoskins

Grantham Institute, Imperial College London,

South Kensington Campus, London, UK

D. Hassell

Met Office, Hadley Centre (Reading Unit) Meteorology

Building, University of Reading, PO Box 243,

Earley Gate, Reading, Berkshire RG6 6BB, UK

K. Hodges

Environmental Systems Science Center,

University of Reading, Reading, UK seen in the model when it is forced with low-resolution SSTs. In addition there are several clear mesoscale responses to increased spatial SST resolution, with surface heat fluxes and convective precipitation increasing by 10 $20 \%$ along the Gulf Stream SST gradient.

Keywords Atmosphere ocean interaction - Gulf Stream

\section{Introduction}

The North Atlantic storm track begins over the east coast of North America where strong surface temperature gradients contribute to high baroclinicity, making the region favourable for cyclogenesis. In winter in particular, a large component of the temperature gradient is due to the contrast between the cold land and the relatively warm ocean. In addition, the sea surface temperatures (SSTs) just off the coast are characterised by very large gradients associated with the Gulf Stream, which can frequently be of the order of a few degrees Kelvin per $100 \mathrm{~km}$, and also by unusually large temporal variability. It is often assumed that these strong SST gradients are important contributions to the overall baroclinicity and hence storm growth, and some recent studies have indeed supported this (e.g. Inatsu et al. 2002; Nakamura et al. 2004; Brayshaw et al. 2008; Nakamura et al. 2008). There has been much speculation that SST variations in the Gulf Stream region may lead to variations in baroclinicity which could affect storm growth and so influence the whole of the storm track (e.g. Xie 2004; Minobe et al. 2008).

It has been known for some time that in the Gulf Stream region temperature variations occur on much finer scales than those represented on coarse grids with resolutions of a few degrees, such as those used in current general 
circulation models (GCMs) and reanalyses. The low resolution of these models compared to those used for numerical weather prediction is often cited as one of the major sources of structural uncertainty inherent in climate projections. A key question is whether the storm track shows any sensitivity to the fine-scale structure of the SSTs, and the associated large local gradients. Is the largescale SST distribution sufficient to represent the storm track well, or are current GCMs missing a vital link between the ocean and the atmosphere?

Doyle and Warner (1993) first presented a case study of a midlatitude storm for which a numerical forecast was indeed sensitive to the resolution of the SSTs used. Increasing the SST resolution improved both the position and intensity of the storm when compared to observations. Similar cases have since been noted by Xie et al. (2002) and Thiébaux et al. (2003). Yamamoto and Hirose (2007) found that the use of eddy-resolving SST data improved the simulation of a storm in their case study, though interestingly this was attributed to unrealistically strong SST gradients in the optimum interpolation SSTs which lacked mesoscale ocean eddies.

A related question is whether the storm track is sensitive to the temporal frequency at which SSTs are prescribed in an atmosphere model, though this has received little, if any, attention in the literature. This paper investigates both of these questions by using a regional atmosphere model (RAM) to simulate the North Atlantic storm track. A RAM is a limited area numerical model which simulates just a small region of the globe, and thus requires continuous forcing of the prognostic variables at the lateral boundaries. The basic approach used here is to run the RAM for periods of 15-20 years, driven at the lateral boundaries with reanalysis data and at the surface with a variety of different SST datasets at different spatial and temporal resolutions. The use of a regional, as opposed to global model allows us to perform relatively long integrations at high resolution, so that the dynamics of mid-latitude cyclones are represented well. In addition, the use of a regional model ensures that any change in the storm track is a local response to the imposed forcing. RAMs are usually used to downscale the information generated by GCMs, in which case a small domain is chosen for the RAM so that the large scale flow is well constrained by the boundary conditions. Here, however, we take a different approach. Since the aim is not to downscale the global data, but to investigate the sensitivity of the synoptic and large-scale flow to the SSTs, we deliberately use a large domain for the RAM simulations, as shown in Fig. 1. This means that the large-scale flow predicted by the model is able to drift from that specified by the boundary conditions (Jones et al. 1995), so that changing the SSTs could potentially result in changes to the large-scale flow within the domain. A similar

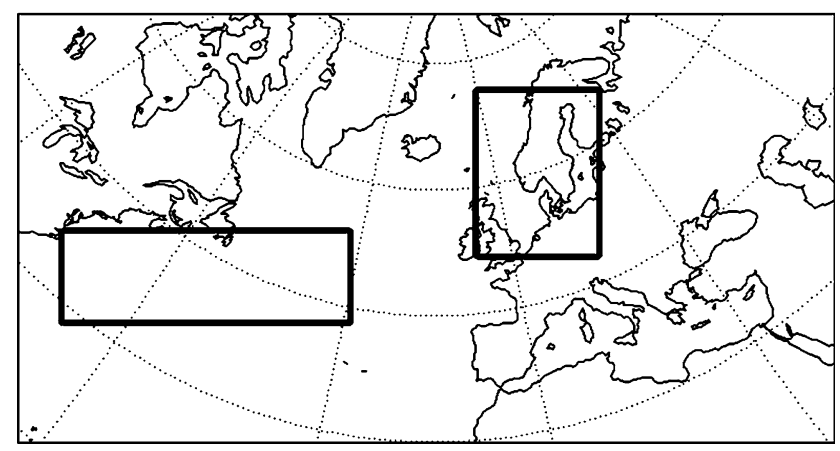

Fig. 1 The model domain, together with locations of various regions used in the text, namely Northern Europe and Gulf Stream

approach was used successfully by Semmler et al. (2008) to investigate the storm sensitivity to a uniform increase in SST.

Regional atmosphere models are most successfully used when there is strong high-resolution forcing within the domain which is absent in the lower resolution GCM, as is often true in the presence of detailed orography. The finescale structure of SST fronts such as the Gulf Stream is another potential example of important high-resolution forcing. The assessment of the importance of this forcing on climate timescales has been made possible by recent developments in historical SST datasets. For some years the best historical SST dataset for many climate applications has been that of Reynolds et al. (2002), R02 hereafter. This dataset was generated by combining AVHRR ${ }^{1}$ satellite and in situ SST observations using optimal interpolation to fill data gaps, providing a full gridded field on a $1^{\circ}$ grid. Within the last 10 years the development of microwave satellite sensors has provided SST observations of much higher effective resolution than previously available (Wentz et al. 2000). These have confirmed the dominance of fine-scale structure in regions such as the Gulf Stream (Chelton and Wentz 2005), and show that SST gradients in these regions are generally significantly stronger than those seen in the R02 data.

The fine-scale structure seen in satellite observations led Thiébaux et al. (2003) to attempt a re-processing of the historical AVHRR data at higher resolution, with considerable success. This showed that the effective resolution in the R02 data was limited by the correlation length scales used in the optimal interpolation, which were of the order of $600-800 \mathrm{~km}$. This in turn led Reynolds et al. (2007), R07 hereafter, to generate a new version of their dataset using variable length scales, which were as low as $100 \mathrm{~km}$ in regions such as the Gulf Stream. This version contains a level of fine-scale detail which, while lower than that in

\footnotetext{
${ }^{1}$ Advanced Very High-Resolution Radiometer.
} 
Fig. 2 Snapshots of the SST on 15 January 1985 , as represented in the datasets used here. a ERA40 SST. b Reynolds07 SST. c Reynolds07 SST smoothed. d Reynolds07 SST smoothed-ERA40 SST. Contours are every $1 \mathrm{~K}$ in a-c and every $0.5 \mathrm{~K}$ in d a

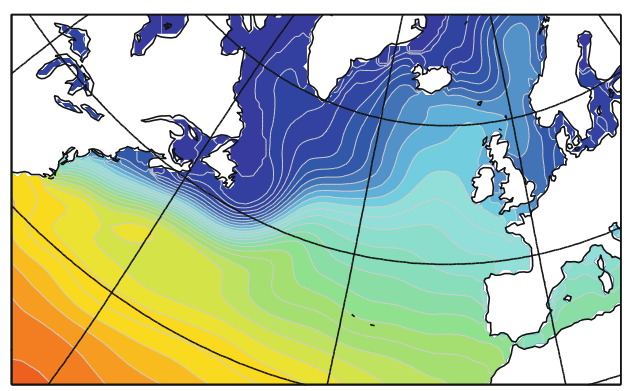

b

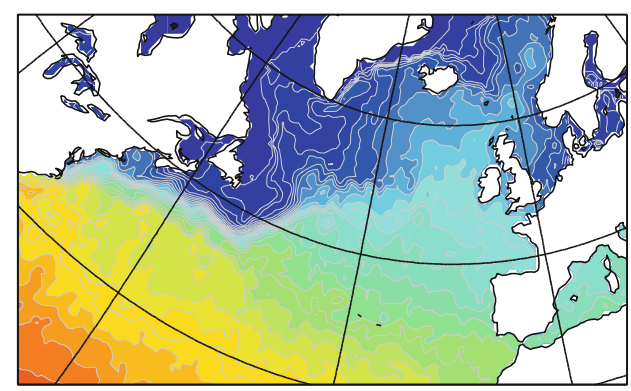

C

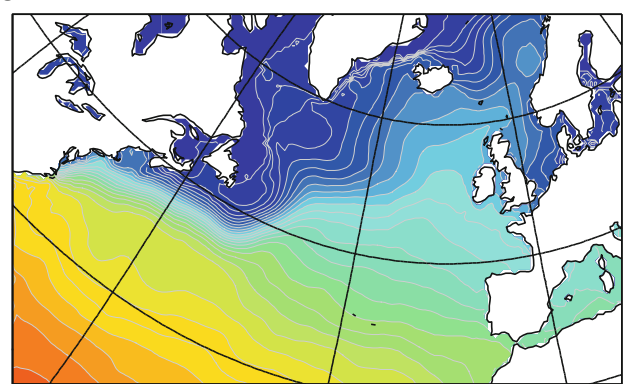

d

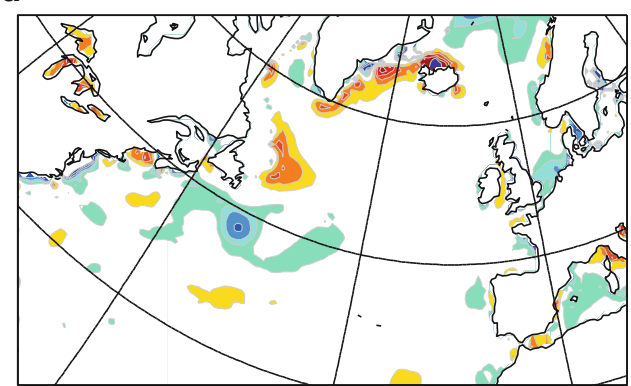

microwave data, is a considerable improvement on the earlier version. The reprocessing of historical observations back to 1985 gives for the first time a relatively long time series of truly high-resolution SST fields. As an example of the effective resolutions, Fig. $2 \mathrm{a}$ and $\mathrm{b}$ show snapshots of the R02 (the ERA-40 version) and R07 SSTs, respectively, as seen on the model's $50 \mathrm{~km}$ grid.

We have performed three different comparisons. First we assess the importance of spatial resolution in experiments using the R07 data at full resolution and then smoothed to a resolution similar to that of R02. Then we assess the importance of temporal resolution in both of the SST datasets. Using the R02 data, we compare two experiments, one at monthly and the other at weekly frequency. Finally we compare experiments using daily and monthly versions of the R07 data. While the emphasis is on SSTs, especially in the Gulf Stream region, sea-ice is also applied as a lower boundary condition. In the spatial resolution experiment the sea-ice is unchanged, but in the temporal resolution experiments it was treated in the same way as the SST field in order to ensure consistency between sea-ice and SST fields.

\section{Methodology}

\subsection{The model}

The model used here is HadRM3p. This is the regional version of the Hadley Centre's third generation atmosphere model HadAM3 (Jones et al. 1995), with modifications to some physical parameterisations as described in Jones et al. (2004). The model uses a regular $0.44^{\circ}$ latitudelongitude grid, which is rotated so that the region of interest lies over the grid's equator, giving a roughly uniform grid size of $50 \mathrm{~km}$. The model has 19 levels in the vertical. The lateral boundary conditions are applied continuously over a four point rim by relaxing the model variables towards the boundary data. The boundary conditions are specified every $6 \mathrm{~h}$, with intervening values obtained by linear interpolation in time. Within the four point rim, the weighting given to the boundary conditions is $1,0.75,0.5$ and 0.25 , with the values decreasing towards the interior of the domain. No relaxation is applied in the subsequent four cells, but the orography is smoothed in these to ensure a gradual transition to higher resolution. In all the experiments described here the lateral boundary conditions are derived from the ERA-40 reanalysis (Uppala et al. 2005). The model is integrated over a large region extending from the east coast of North America to just east of the Mediterranean. The region, excluding both the four point rim and the additional four points with smoothed orography, is that shown in Fig. 1.

\subsection{Spatial resolution experiment}

This experiment uses the high resolution R07 SSTs which contain length scales of around $100 \mathrm{~km}$ in the Gulf Stream region. This data is the AVHRR-only version described by R07 and was obtained from the NOAA National Climatic 
Data Center. At the time of writing it was available from 1985 onwards. The data were first bilinearly interpolated onto the model grid. Coastal cells could often not be defined by this method, and values for these were chosen by averaging the SST in neighbouring ocean cells. Sea-ice concentrations were also bilinearly interpolated onto the model grid. This version of the R07 data suffers from a small level of noise near the coasts in the sea-ice field (see R07), and this necessitated some further pre-processing. Firstly the coastal cells undefined by the interpolation were defined using the ERA-40 data. Secondly any ice cells adjacent to a coast, but outside of the climatological maximum ice extent derived from ERA-40, were removed. The model was then integrated over the period January 1985-November 2000, using ERA-40 SSTs from 1984 to run the spin-up year. This gives 16 years of model output, but only 15 complete winter seasons. This run will be referred to as HIGH-RES.

The impact of SST resolution is assessed by comparing HIGH-RES to another run, called LOW-RES, which is identical except that a smoothed version of the SST data is used as a boundary condition. The smoothing was achieved by replacing each SST value by an average of the values in a $9 \times 9$ square of cells (roughly $450 \times 450 \mathrm{~km}$ ) centred on the cell in question. Close to the coastlines this results in unrealistic values, so successively smaller squares were used for averaging, to the point where cells adjacent to the coastline are unchanged. The sea-ice data for this run is identical to that in HIGH-RES, so that this experiment compares the effect of SST resolution in isolation. In both runs the ERA-40 SST and sea-ice values are used in the model's four point boundary rim, in order to maintain consistency with the lateral boundary conditions. Over the next four cells the high-resolution data is introduced gradually with weightings of $0.25,0.5,0.75$ and 1 .

Figure $2 \mathrm{c}$ shows the effect of this smoothing on the HIGH-RES snapshot in Fig. 2b. The effective resolution has been reduced to a similar level to that seen in the R02 data in Fig. 2a. To characterise the resolutions of the different datasets, the 95th percentile of the horizontal temperature gradient over the Gulf Stream region (shown in Fig. 1) was calculated each week. The gradients were calculated using simple differences between neighbouring grid cells. Figure 3 shows the distribution of the 95th percentile using all the data from 1985 to 2000 . The gradients in the R07 SSTs are almost twice as strong as those in the R02 SSTs, in agreement with Chelton and Wentz (2005), and they also exhibit a larger variance. The range of gradients seen in the smoothed version of R07 is very similar to that in the ERA-40 data. The smoothed SST field in Fig. 2c clearly still exhibits a strong local SST gradient across the Gulf Stream. The aim of this study is not to determine the effect that the presence of the Gulf Stream
Weekly 95pc of gradient over Gulfstream: 1985-2000

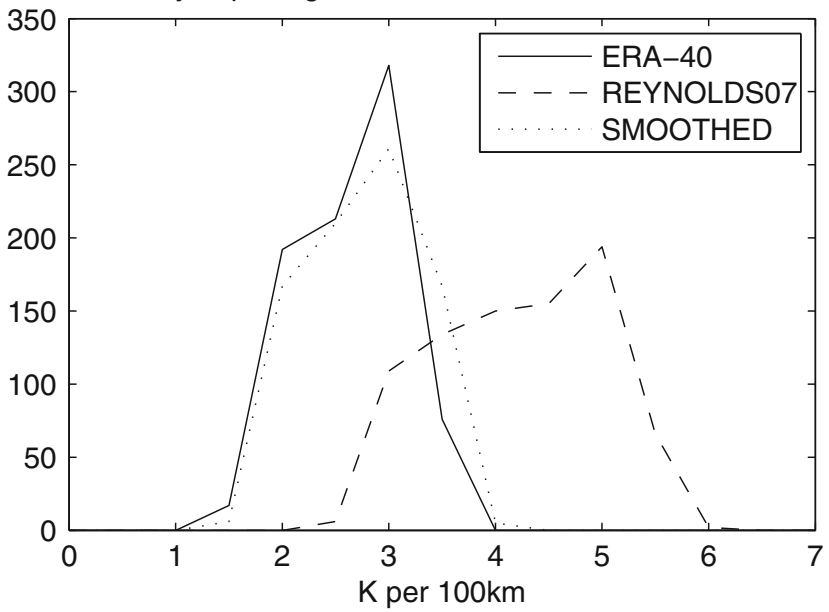

Fig. 3 Weekly distributions of the 95th percentile of the horizontal temperature gradient over the Gulf Stream region (shown in Fig. 1), using all seasons over 1985-2000

has on the atmosphere, but rather to determine the consequences of not resolving the Gulf Stream properly in numerical models. The atmospheric response in these experiments is therefore expected to be smaller than in experiments such as those of Nakamura et al. (2008) where the ocean front is removed in its entirety.

The smoothed R07 and ERA-40 SSTs are differenced in Fig. 2d. This shows that there are differences between the two datasets on fairly large scales, even though they are both derived from the same observations. For this reason, model runs driven by R02 and R07 SSTs are not directly comparable, and in order to cleanly assess the effect of resolution this spatial resolution experiment uses only R07 SSTs.

\subsection{Temporal resolution experiments}

The importance of temporal resolution is assessed using both SST datasets. First we use the low resolution ERA-40 SSTs and assess the effect of upgrading from monthly to weekly resolution. The ERA-40 SSTs are derived from the R02 dataset. The SSTs are given on a $1.125^{\circ}$ grid but the effective resolution of the data is much coarser, as described above. These data are at weekly resolution from December 1981 onwards. Model runs were performed for 19 years from December 1981 to November 2000, after a 1 year spin-up period using monthly ERA-40 SSTs. Two runs were performed, one using monthly and the other weekly SSTs, and these will be referred to as MONTHLY and WEEKLY. The monthly data were derived from the weekly data using the AMIP II method of Taylor et al. (2000) which preserves the monthly mean values under linear interpolation. In both cases the SSTs were imposed 
continuously, using values linearly interpolated in time between the two nearest data points. The sea-ice was also derived from ERA-40 and was treated in exactly the same way as the SST data, so was applied at monthly and weekly frequencies in the two runs, respectively.

To illustrate the difference between the weekly and monthly SSTs, Fig. 4a shows the standard deviation of this difference field. This was calculated on the model grid using daily data generated by interpolating linearly in time. Data from all seasons over the period 1985-2000 were used. Standard deviations of $0.4 \mathrm{~K}$ are seen in the Gulf Stream region, and higher values in the sea-ice regions further north, so there is sub-monthly variability in the SSTs which may be large enough to influence the storm track. An example one-point regression map of the difference field is given in Fig. 4c, which shows that the submonthly variations are of a reasonably large scale, so that synoptic scale SST gradients are influenced by this variability. Since the effective spatial resolution of this data is limited, as described above, it is not surprising that submonthly variations have this scale.

The second temporal resolution experiment uses the R07 data, comparing the HIGH-RES run to a run which uses a monthly version of the high-resolution SSTs and sea-ice (referred to as MONTHLY_HI), but which is otherwise identical. The SSTs for this run were created from the daily SSTs using the AMIP II method as above. The submonthly variations in the R07 data are characterised in Fig. $4 \mathrm{~b}$ and $\mathrm{d}$ in the same way as for the R02 data. The regression map shows that, as expected, the variations have a much smaller spatial scale than in the R02 data. This confirms that the sub-monthly variations in R02 have an unrealistically large spatial scale, so it is clearly of interest to assess the sensitivity to temporal resolution using both datasets.

\subsection{Storm track analysis}

The storm track is analysed using the feature tracking software of Hoskins and Hodges (2002) to identify storms as maxima of vorticity at $850 \mathrm{hPa}$. Model winds are output every $3 \mathrm{~h}$ and used to calculate the relative vorticity on the model grid. Since the model resolution is quite high, the vorticity field contains much fine-scale structure such as fronts. In order to reliably identify the synoptic scale systems the vorticity is smoothed to a similar level as in Hoskins and Hodges, who used a T42 spectral truncation on the globe. Standard spectral methods cannot be applied to the regional model output as the domain is not periodic, so a discrete cosine transform was used to transform the vorticity field, following Denis et al. (2002). The filter of Sardeshmukh and Hoskins (1984) was then used to smooth the vorticity, with a power of 0.1 on the $1,000 \mathrm{~km}$ wavelength, and the smoothed data were transformed back onto the model grid. Since vorticity is used to identify features, there is no need to remove a background field from the data before tracking. Vorticity maxima were then identified as cyclones and tracked while applying constraints to ensure the tracks are suitably smooth.
Fig. 4 a Standard deviation of the difference between weekly and monthly ERA-40 SSTs, using all seasons over 19852000. c An example one-point linear regression map of the weekly-monthly difference field, with base point $42 \mathrm{~W}, 47 \mathrm{~N}$. $\mathbf{b}, \mathbf{d}$ as $\mathbf{a}, \mathbf{c}$ but showing the daily-monthly difference using the R07 data

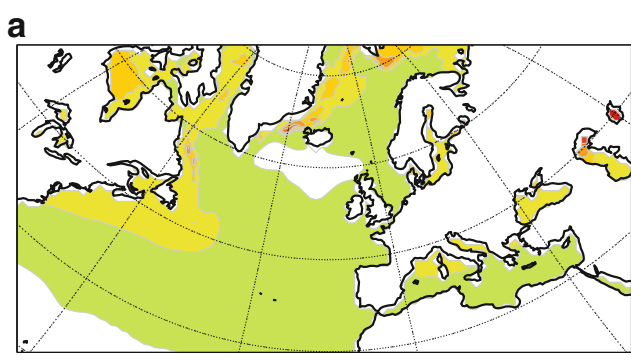

b
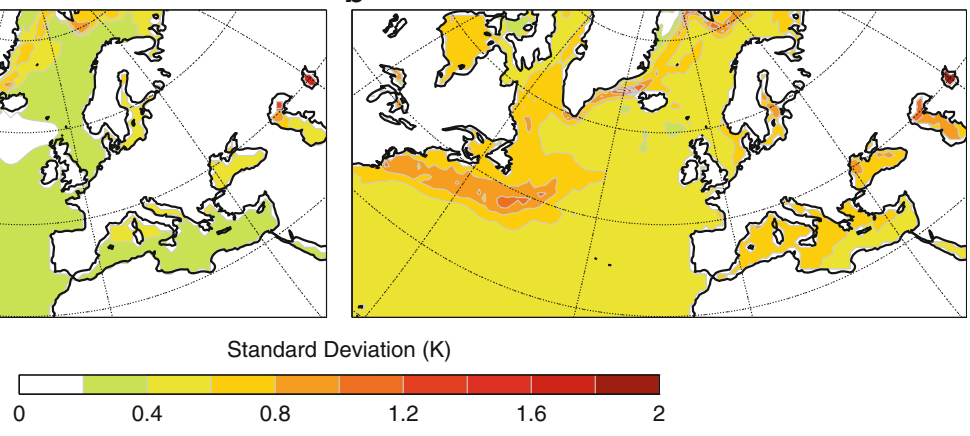

c

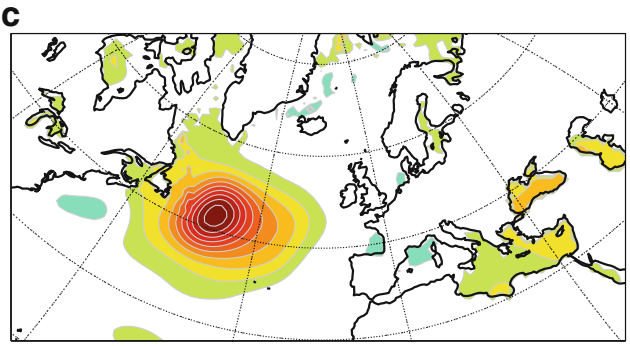

d

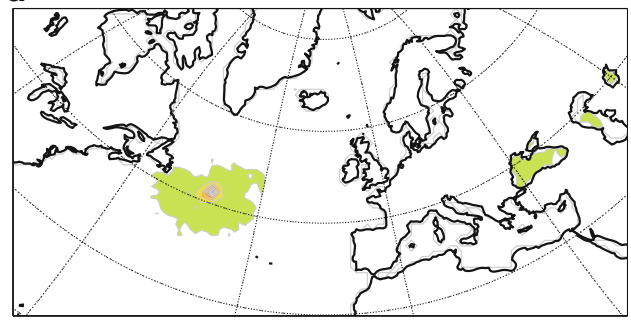

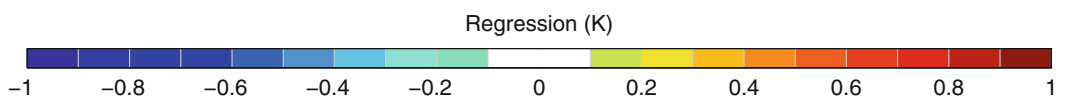


The general position and intensity of the storm track in these experiments is described by the track density, defined as the number of individual cyclone tracks per unit area per season, where the area corresponds to a $5^{\circ}$ spherical cap. The significance of differences in the track density between runs is estimated using the permutation method of Hodges (2008), in which the two sets of cyclone tracks are pooled and then resampled 2,000 times to provide a distribution of track density differences with which to compare the actual difference. Measures of storm activity are invariably noisy, so it is often hard to determine significance. The model runs used here appear to be just long enough to give some significant results for the winter season, but longer runs may be needed to produce clear results for the other seasons. The length of runs is, of course, limited by the availability of observational data.

\subsection{Large-scale changes}

As described above we deliberately used a large model domain to allow the possibility that the large-scale flow may diverge from that prescribed by the LBCs. This could be envisaged, for example, through modifications to the storm track and its associated mean flow forcing. However, even with a large domain, the lateral boundary conditions do partly constrain the large-scale flow, so it is not entirely free to evolve. To quantify this we use an empirical orthogonal function (EOF) analysis to identify large-scale patterns of variability in the interior flow. The driving reanalysis data are then interpolated onto the model grid to create a matching dataset, and the EOF patterns are projected onto these data to generate time series of these patterns in the reanalysis data. These time series are then correlated with the principal components in order to test the agreement between the model and the driving data. When this is performed using daily wintertime data, for all of the leading EOF patterns the resulting correlations are close to 0.7 . This suggests that about $50 \%$ of the large-scale variability in the model domain is constrained by the boundary conditions.

In fact, we have found no significant differences in the mean flow, in patterns of variability, or in the occurrence of blocking regimes in any of the experiments presented here. There are, however, significant differences in the storm track, as described below. In general a change in the storm track would be expected to lead to a change in the largescale flow. In Sect. 4 we give an example of the mean zonal wind field in the model and show that the response to changing SSTs is consistent with the change in the storm track, but that the mean wind response does not pass the significance test. This may be because the storm track changes are subtle, so the lack of any large-scale response simply reflects the weakness of the signal. However, it cannot be ruled out that the lack of a large-scale response is due to the partial control over the large-scale flow by the LBCs.

\section{Storm track sensitivity to SST resolution}

This section compares the storm tracks simulated by the HIGH-RES and LOW-RES runs. Figure 5 shows the track density in winter for the two runs and their difference. Increasing the SST resolution acts to shift the start of the storm track slightly off the coast and over the Gulf Stream SST gradients. The difference between the two runs is only clear over the Gulf Stream region, though even there the difference barely passes the significance test. There are no clear changes downstream. While the storm track changes are subtle, the pattern is robust to the choice of storm track diagnostic. For example, the 2-6-day bandpass filtered sea level pressure variance has been calculated, and the resulting difference pattern (not shown) is similar to that in Fig. 5, if a little noisier. The shift over the Gulf Stream is as might be expected given the importance of SST gradients on the large scale, and is in agreement with Thiébaux et al. (2003), who gave examples of storm forecasts which developed too close to the North American coast when low-resolution SSTs were used. As described in Sect. 1, several studies have shown the potential of SST resolution to influence the path of individual storms. This suggests that models using low-resolution SSTs may overestimate storm activity along the coast of North America. In these models the storm track is likely to be too closely tied to the coastline, and may not be sensitive enough to variations in the SSTs in the Gulf Stream region.

In the case study of Xie et al. (2002), increasing the SST resolution led to an increase in the intensity of the cyclone, so it is of interest to look for changes in growth rate and intensity of storms. Figure 6 shows distributions of the storm intensity and tendency in the Gulf Stream region shown in Fig. 1 in the two experiments. For both quantities this is the maximum vorticity value that each storm attains within this region. The tendency is the absolute difference in vorticity over $3 \mathrm{~h}$, without scaling by the total vorticity. The changes between the two runs are small, and indicate changes in the number of storms of mid-range tendency and intensity. There is no sign of an increase in explosive cyclogenesis, as might have been expected, but this is consistent with Xie et al., as the intensity increase seen there arose because the cyclone continued deepening at the same rate for a longer period.

The storm track differences in the other seasons have also been examined, but the differences are very weak and unclear, so are not shown here. The lack of a significant response in these seasons may be due to the storm track 
850hPa Vorticity Track Density: LOW-RES (DJF 85/86 - 99/00)

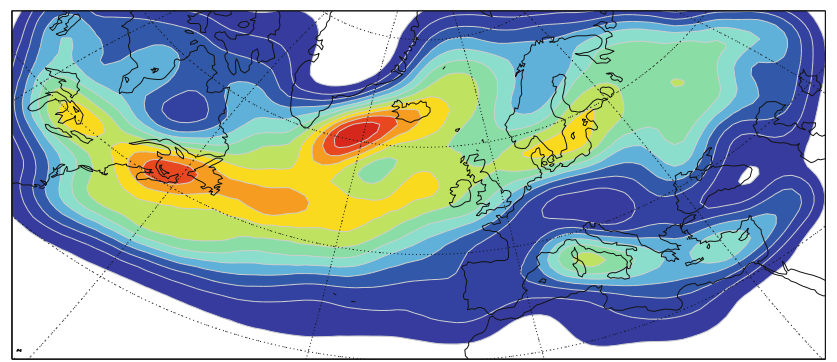

850hPa Vorticity Track Density: HI-RES (DJF 85/86 - 99/00)
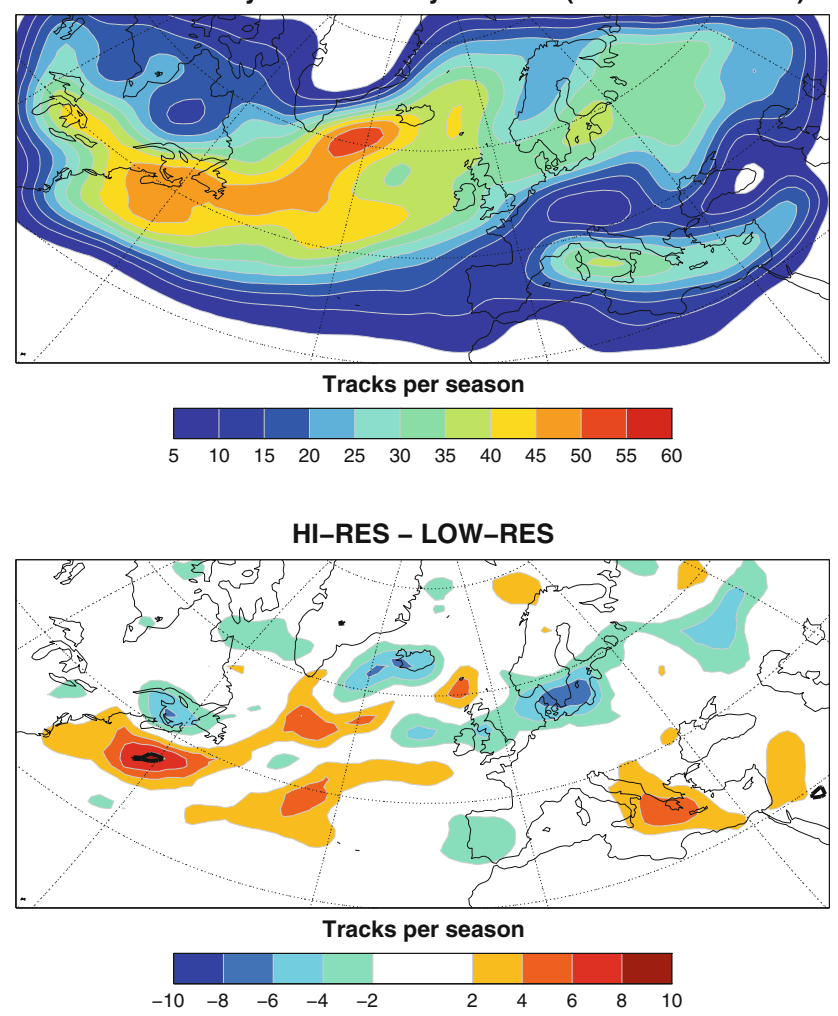

Fig. 5 A comparison of the winter storm track in the LOW-RES and HIGH-RES experiments. The diagnostic is track density, which is the average number of cyclone (vorticity maxima) tracks per season per unit area. Thick black contours enclose regions where the difference is significant at the $95 \%$ level using the permutation test lying further north, so that the interaction with the Gulf Stream SSTs is weaker.

Although our focus is on the storm track changes, it is worth noting that there are several clear mesoscale differences between the HIGH-RES and LOW-RES runs over the Gulf Stream. There is a general increase in the latent and sensible heat fluxes from the ocean to the atmosphere over the Gulf Stream. These increases are of the order of $10-20 \%$ respectively in winter, and as an example the latent heat fluxes and precipitation are shown in Fig. 7. The precipitation in this area is also increased, by around 10-20\% depending on season. The precipitation change is almost entirely convective, rather than large-scale rainfall. This is consistent with a local convective response to the SSTs, though it is not known how realistic this model response is. These mesoscale responses are consistent with those seen in other studies, such as Warner et al. (1990) and Minobe et al. (2008).

There are two possible mechanisms underlying the storm track sensitivity to SST resolution, and it seems likely that both contribute to the response. Firstly, it is possible that the increased SST gradient leads to an increased temperature gradient at low levels in the atmosphere which provides for enhanced baroclinic growth. This mechanism was demonstrated by Kuo et al. (1991) in a case study of a storm growing over the Gulf Stream in a mesoscale model. Convection over the warm side of the Gulf Stream acted to destabilise the atmospheric boundary layer, leading to a strong low-level atmospheric front oriented along the Gulf Stream. The increase of convection along the warm side of the Gulf Stream in Fig. 7 shows that this process also operates in our experiment, but in this case it is not clear that the atmosphere can respond strongly to the increased baroclinicity. The SST fields used here differ mostly on scales less than $500 \mathrm{~km}$, i.e. on scales which are shorter than the atmospheric radius of deformation, so the ability of baroclinic waves to make use of the increased baroclinicity is expected to be limited. Taguchi et al. (2009) have also demonstrated this
Fig. 6 Distributions of the intensity and $3 \mathrm{~h}$ tendency of cyclones in the Gulf Stream region (as in Fig. 1) in the HIRES and LOW-RES experiments
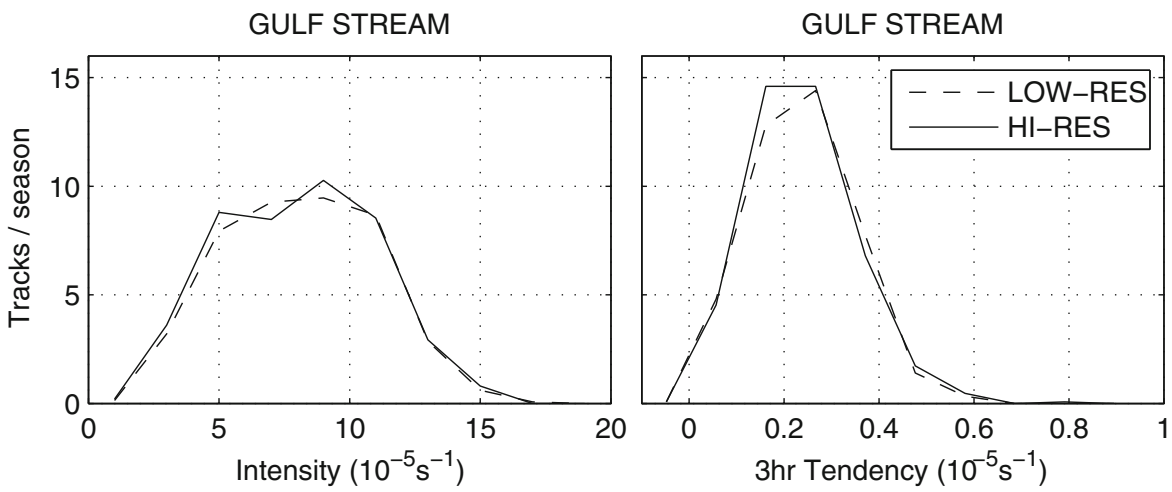
Fig. 7 Comparison of the winter mean total precipitation $(\mathbf{a}-\mathbf{c})$ and surface latent heat flux $(\mathbf{d}-\mathbf{f})$ between the LOWRES and HI-RES experiments. a Low-Res SSTs. b Hi-Res SSTs. c Hi-Res-Low-Res. d Low-Res SSTs. e Hi-Res SSTs. f Hi-Res-Low-Res. Black contours enclose regions which are significantly different at the 95\% level using the two-sided small-sample lookup test of Zwiers and von Storch (1995) on the individual winter means a

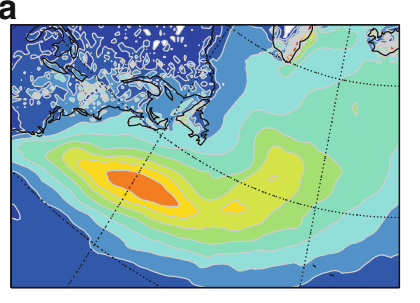

b

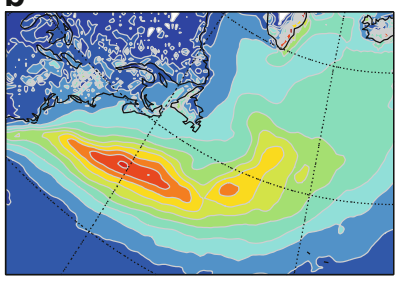

DJF mean PRECIP (mm/day)
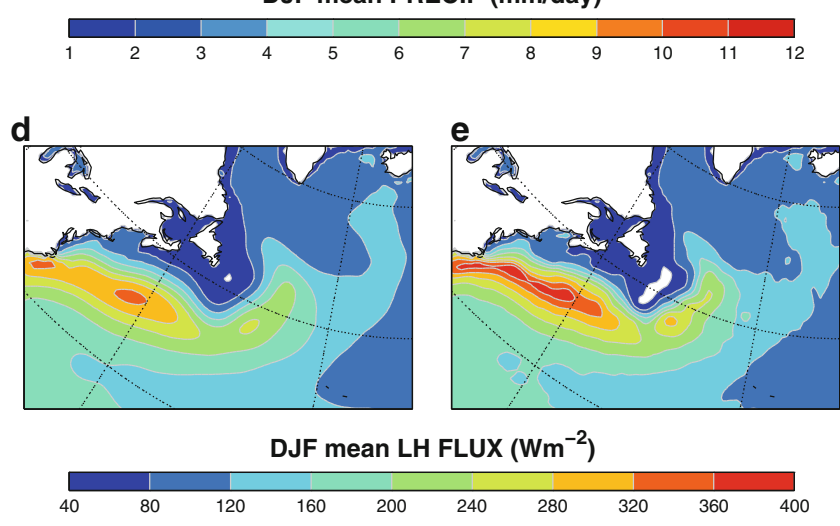

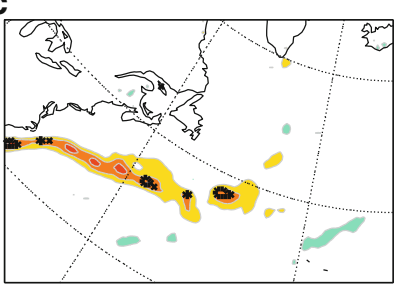

PRECIP Difference ( $\mathrm{mm} / \mathrm{day})$

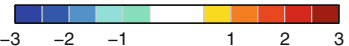

f

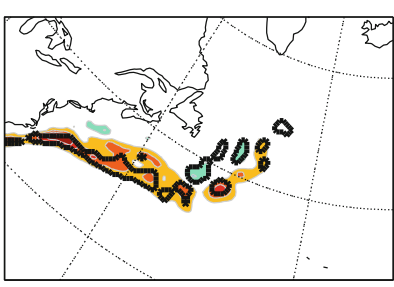

LH FLUX Difference $\left(\mathrm{Wm}^{-2}\right)$

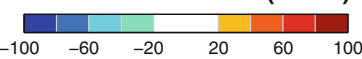

mechanism acting over midlatitude ocean fronts in experiments similar to those presented here. However, the SST fields in their experiments were smoothed more strongly than those used here.

Secondly, the increased surface heat fluxes over the Gulf Stream act to warm and moisten low-level air which could provide additional latent heating to storms during their subsequent growth (Kuo et al. 1991; Hoskins and Valdes 1990). Of all the incoming small perturbations, the ones which pass over the Gulf Stream will be enhanced by this heating and this could also explain the shift of the storm track onto the SST gradient.

\section{Storm track sensitivity to SST frequency}

First we compare the results of the 19-year MONTHLY and WEEKLY simulations. Figure 8 shows the track density averaged over the 19 winter (DJF) seasons in the MONTHLY and WEEKLY runs, and their difference. Using weekly SSTs results in an increase in track density along the east coast of North America, where the variance in Fig. 4 is large. There is also a significant increase in storm activity throughout the central part of the storm track, extending well into Scandinavia. There are weak reductions in track density to the north and south of this, so the signal might be partly that of a focusing of storm paths. In contrast, there is a slight weakening of the Mediterranean storm track, even though the sub-monthly variance is also quite large there (Fig. 4a). This could be due to the focusing of Atlantic storm activity towards Northern
Europe, which would reduce the seeding of Mediterranean storms by the remnants of Atlantic storms.

As before, the 2-6-day bandpass filtered sea level pressure variance shows a similar response, so the result is not sensitive to the method of storm track diagnosis. Figure 9a shows the distribution of storm intensities over the Northern European region shown in Fig. 1 in the two simulations. This shows that the increase in storm activity over Scandinavia is due to an increase in moderate to high intensity (but not extreme) storms.

As an example of the simulation of the large scale flow in the model and its response to the change in SSTs, Fig. 10 shows the winter-mean zonal wind at $850 \mathrm{hPa}$. The top panel shows the difference between the MONTHLY simulation and ERA-40. This difference is small in magnitude, generally not passing the pointwise significance test, but has a large-scale structure which is consistent with the model climate being slightly too zonal, which is a problem shared by many models. The wind response to the change in SSTs is shown in the lower panel. Again, the response is small and does not pass the significance test, as described in Sect. 2.5. However, the pattern of change is consistent with the storm track changes in Fig. 8, since the increased storm activity is expected drive stronger lowlevel zonal winds, especially at the downstream end of the storm track (e.g. Hoskins et al. 1983).

Storm tracking has been performed for all seasons, though of course the storm track is at its strongest in winter. In the other seasons there are many weak systems which obscure the signal. There are some consistent differences between the two runs in the other seasons, in that 

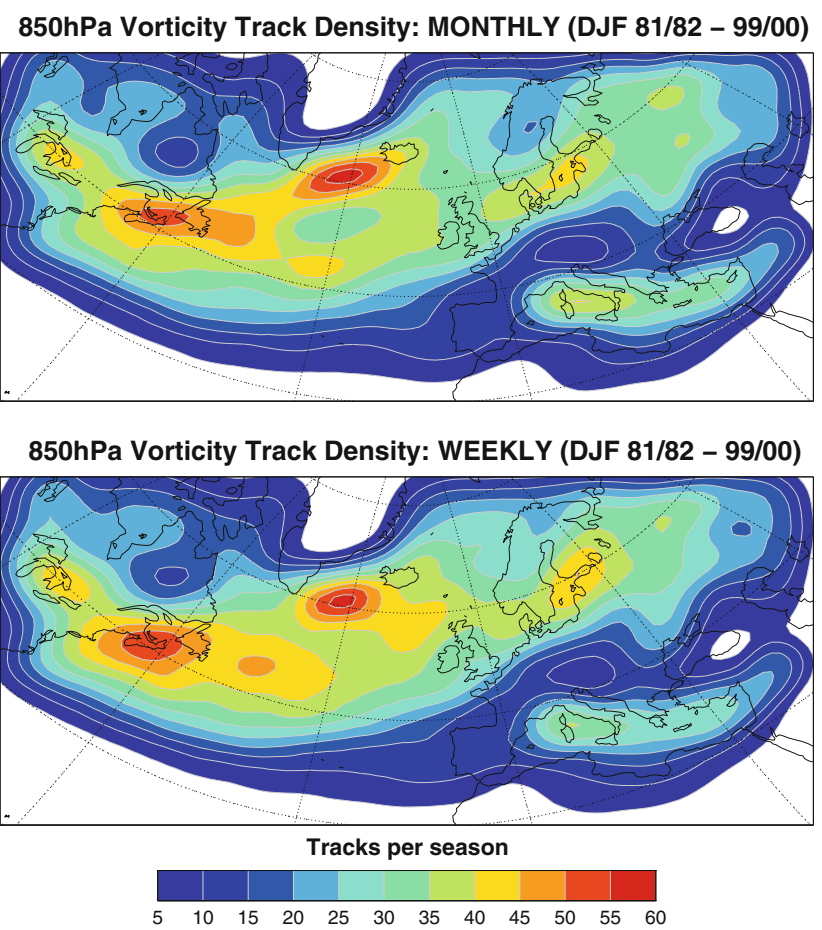

WEEKLY - MONTHLY

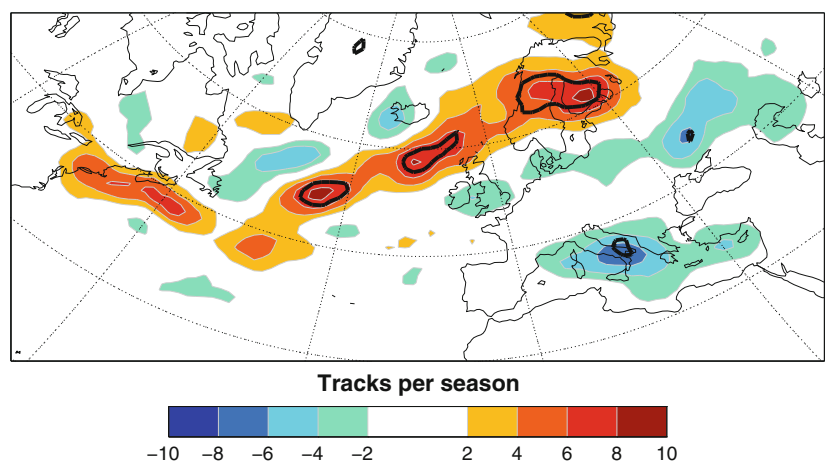

Fig. 8 Winter cyclone track densities, as in Fig. 5, but comparing the MONTHLY and WEEKLY SST experiments

they all share the winter signal of an increase in storm activity extending north of the UK and into Scandinavia, but the pattern is very weak and does not pass the significance test. The pattern becomes clearer if the weaker
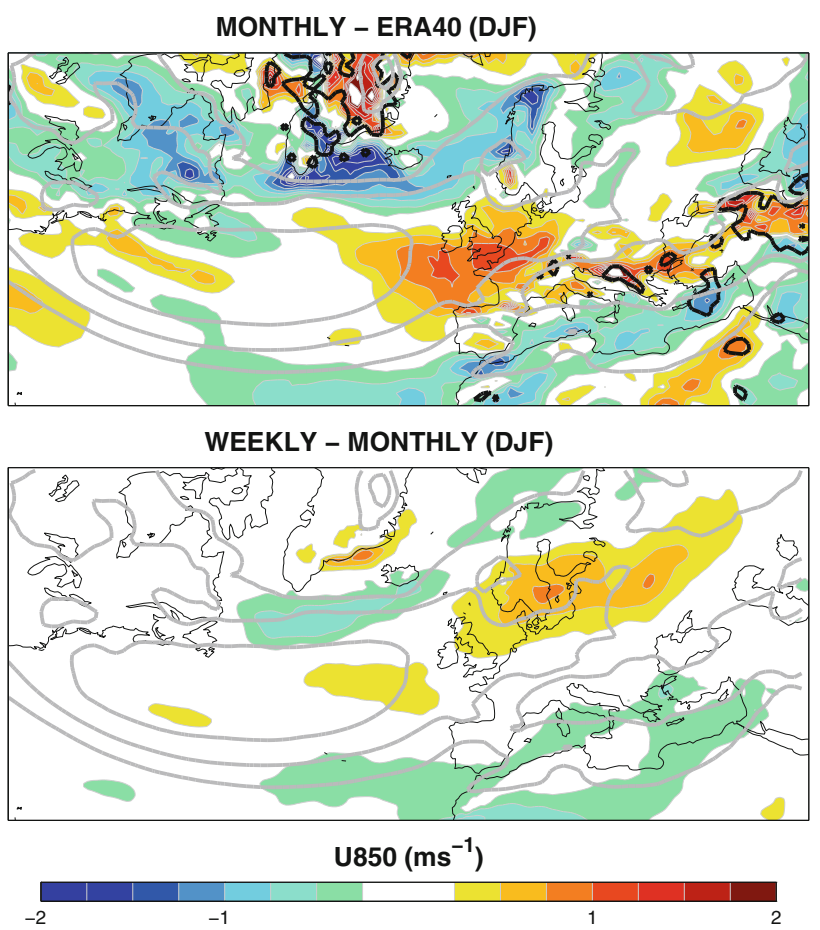

Fig. 10 Differences in the winter-mean zonal wind at $850 \mathrm{hPa}$. The mean field from ERA-40 is overlaid in thick grey contours at 3,6 and $9 \mathrm{~ms}^{-1}$ for reference. Black contours enclose regions which are significantly different at the $95 \%$ level using the two-sided smallsample lookup test of Zwiers and von Storch (1995) on the individual winter means

systems are excluded, for example by restricting attention to only the top $50 \%$ of storms (by intensity) in each season. However, even then the significance test is not passed, so longer simulations would be required to decide if there is a significant response.

We now compare the storm tracks in the MONTHLY_HI and HIGH-RES simulations in Fig. 11. This comparison is quite similar to that between the MONTHLY and WEEKLY runs using the R02 data, with an increase in storm activity over the Gulf Stream, although here the response over northern Europe more resembles a northward shift of activity, with an increase in storm tracks in the
Fig. 9 Distributions of cyclone intensities over the Northern European region shown in Fig. 1. For each cyclone the maximum intensity reached within the region is used. a Comparison of the MONTHLY and WEEKLY runs. b Comparison of the MONTHLY_HI and HI-RES runs

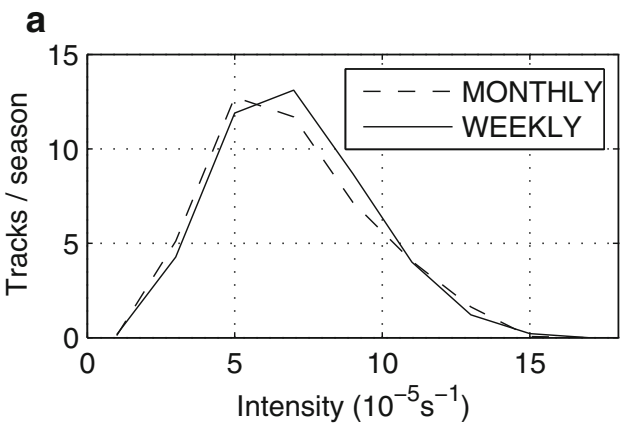

b

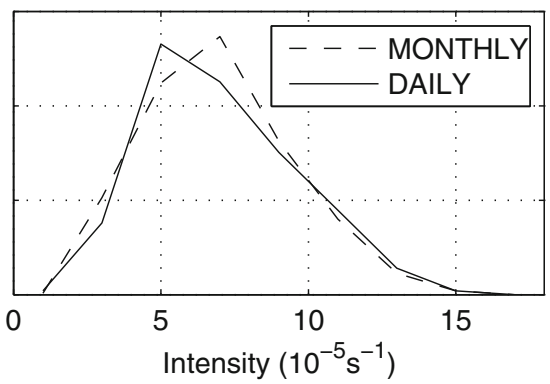


Norwegian Sea and a decrease over the southern Baltic. The decrease of activity over the Baltic dominates the northern European signal, as shown in Fig. 9b. Again, this figure shows that the changes are not simply due to weak systems. The storm track changes in the other seasons are generally very weak and do not pass the significance test. As before, the winter changes are subtle, but the similarity of the responses using the two very different SST datasets suggests there is a real physical sensitivity, and that the sensitivity shown in the experiment with R02 SSTs is not simply due to the unrealistically large scale of sub-monthly variations in that data.

We cannot identify the mechanism behind the storm track sensitivity to SST frequency, but there are several

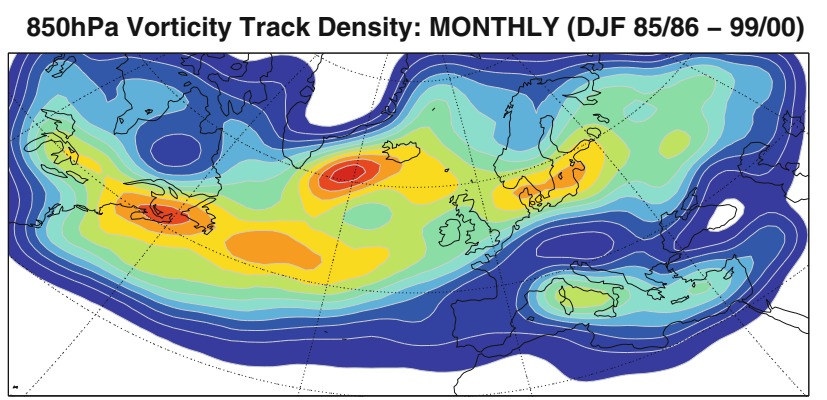

850hPa Vorticity Track Density: DAILY (DJF 85/86 - 99/00)
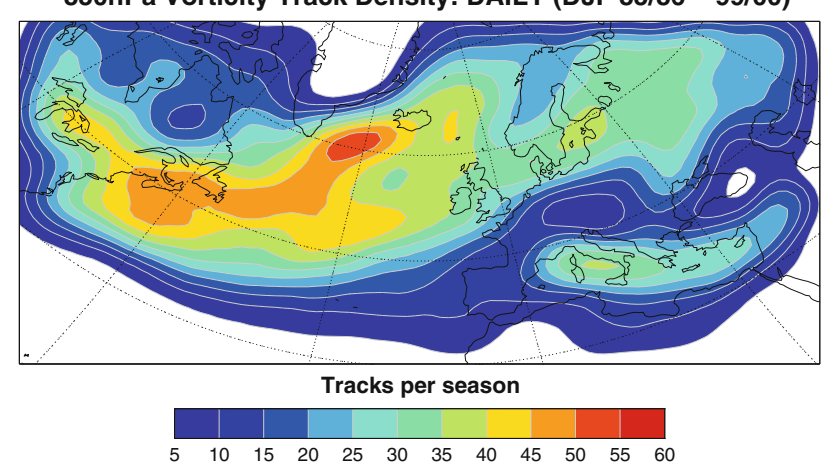

DAILY - MONTHLY

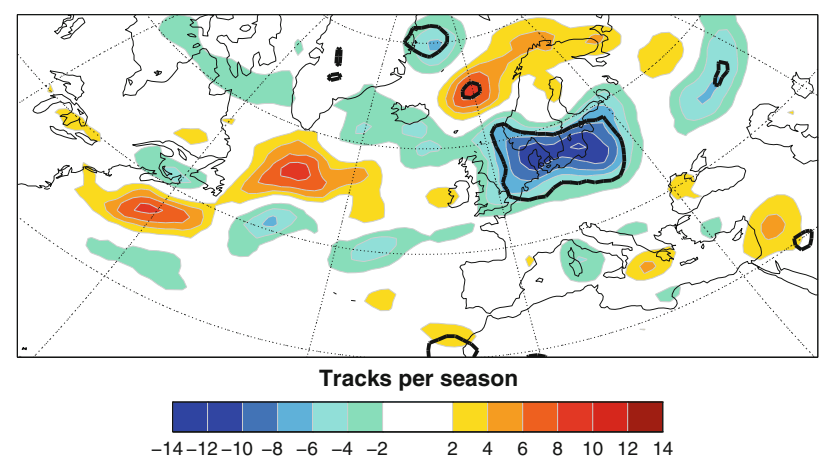

Fig. 11 Winter cyclone track densities, as in Fig. 5, but comparing the MONTHLY_HI and HIGH-RES SST experiments possibilities. The temporal smoothing implicit in the monthly data will mean there are fewer periods of unusually large SST gradients, and hence baroclinicity. However, this mechanism might be expected to have a smaller influence when using the high-resolution SSTs (Fig. 4). Note that the extreme gradients in the Gulf Stream region do not appear to be affected by the temporal averaging (the analysis of Fig. 3 was repeated using the monthly ERA-40 data, and the distribution is virtually unchanged). Alternatively, with sub-monthly SSTs there will also be an enhanced variance of the air-sea temperature contrast, which could affect the latent and sensible heat fluxes. It is, of course, possible that enhanced sea-ice variance is responsible, although given the location of the storm track signal this seems unlikely.

Some contribution to the storm track response may come from the nonlinearity of the low-level baroclinicity with respect to the surface temperature. The baroclinicity can be expressed as the Eady growth rate

$\sigma=0.31 f\left|\frac{\partial \mathbf{v}}{\partial z}\right| \frac{1}{N} \quad$ where $N=\sqrt{\frac{g}{\theta} \frac{\partial \theta}{\partial z}}$.

(See Hoskins and Valdes 1990 for more details). This shows that in the lower troposphere, $\sigma$ is directly related to the surface potential temperature $\theta_{s}$ through the static stability $N$. This relationship is nonlinear, with

$\sigma \propto\left(\theta_{t}-\theta_{s}\right)^{-\frac{1}{2}}$

where $\theta_{t}$ is the potential temperature at some level above the surface. This nonlinearity means that an increase in surface temperature will affect the Eady growth rate more than an equivalent decrease, so an increase in the variance of SSTs will lead to an increase in the mean baroclinicity. Using the climatological field at $700 \mathrm{hPa}$ for $\theta_{t}$, we have calculated the change in this factor in changing from the MONTHLY to WEEKLY SSTs. This gives increases in low-level baroclinicity of 5\% over the Gulf Stream and in the vicinity of the ice edge, so this effect does seem likely to contribute to the observed storm track response. The effect may even be larger since reducing the static stability enables growing baroclinic waves to fill the depth of the troposphere more easily, resulting in waves of longer horizontal wavelength which have larger amplitudes at upper levels and extract more energy from the background flow (Simmons and Hoskins 1978).

\section{Comparison to ERA-40}

The results presented above show that the storm track is sensitive to both the temporal and spatial resolution of SSTs. It is of interest to compare the storm track in these 
Fig. 12 Comparison of the winter cyclone track density in various experiments with that given by ERA-40. For this figure, all tracking was performed on six-hourly data over the common period 1985/ 1986-1999/2000
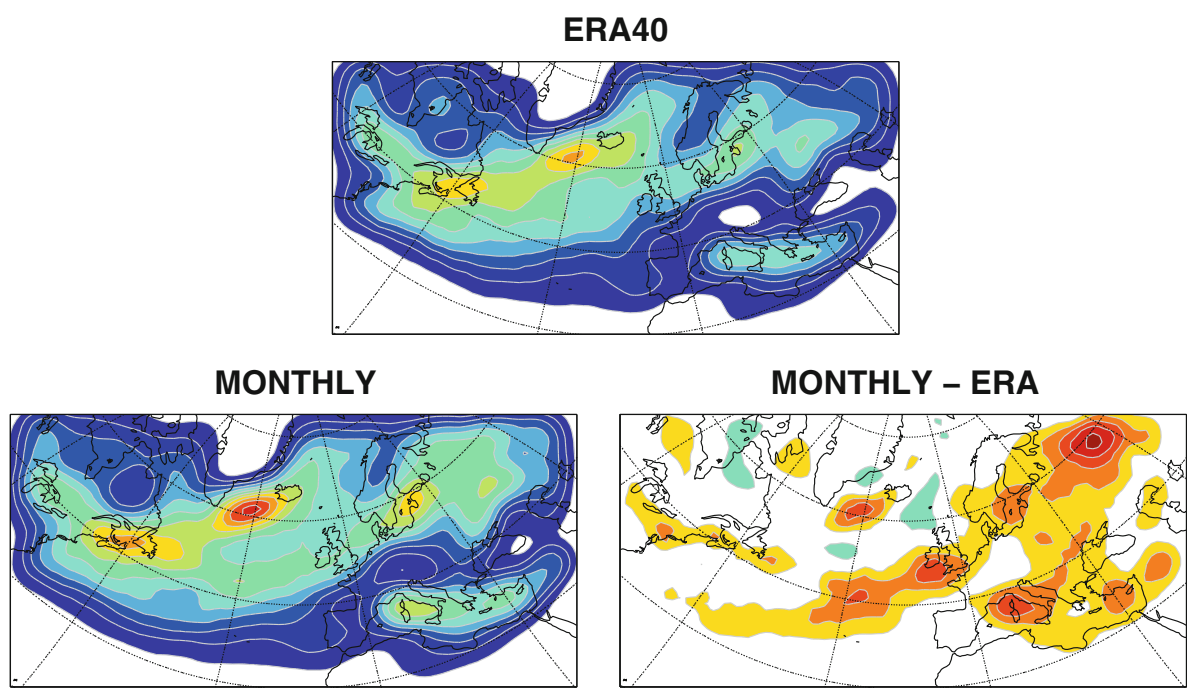

WEEKLY

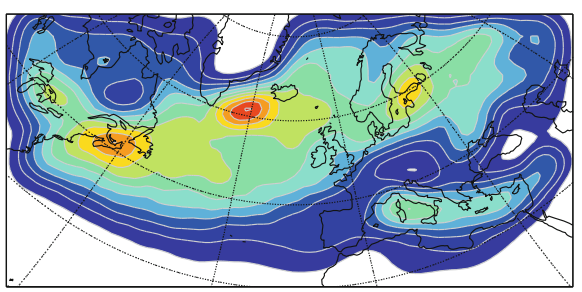

HIRES

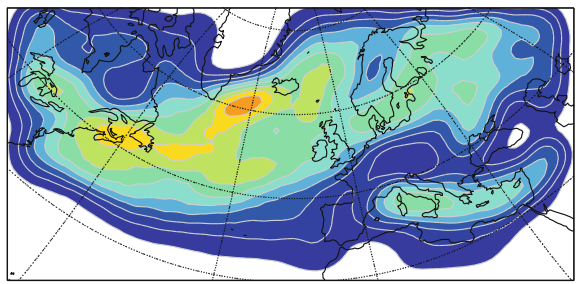

TRACK DENSITY

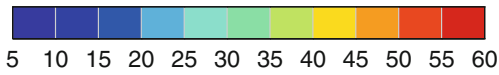

WEEKLY - ERA

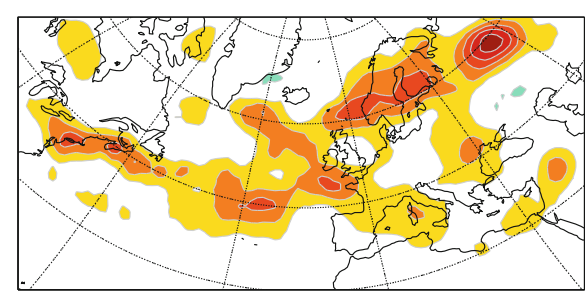

HIRES - ERA

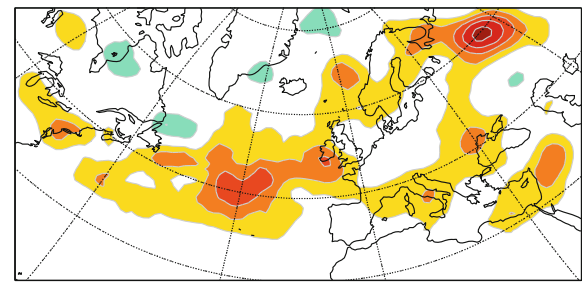

TRACK DENSITY

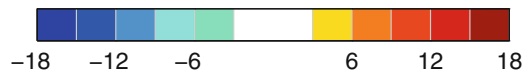

simulations with that in the ERA-40 data. To generate storm track statistics for the ERA-40 data, the winds were first interpolated onto the model grid using linear interpolation. The vorticity was calculated and then this was smoothed using the discrete cosine transform described in Sect. 2.4. This smoothing ensures that features are tracked at the same resolution in both model and reanalysis data, despite the differing resolutions of the original data. The ERA-40 data are only available at six hourly intervals, so for this comparison the model data were sub-sampled to six hourly resolution before tracking.

The results of this comparison are shown in Fig. 12 for the track density in winter for some of the model runs. This shows that the model simulation of the general storm track structure is good in all cases. As before, the differences between the different model runs are quite subtle. Using the R07 SSTs, as in the HI-RES experiment, has reduced some of the biases (i.e. the differences from ERA-40) seen when using the R02 SSTs. For example the MONTHLY, and in some cases the WEEKLY runs show overestimated storm activity near the southern tip of Greenland, along the coast of North America and in the Mediterranean, and these biases are reduced in the HI-RES run. However, the model shows a tendency to overestimate storm activity along the southern flank of the storm track, and this tendency is not reduced. It may seem paradoxical that using R07 SSTs would reduce the model bias when the ERA-40 reanalysis itself was produced using R02 SSTs. This presumably reflects the dominance of the atmospheric observations over the SSTs in determining the storm activity in the reanalysis. Changing from MONTHLY to WEEKLY SSTs has in some ways worsened the agreement 
between the model and the observations. This may be because, as described in Sect. 2.3, the sub-monthly SST variance in the R02 dataset has an unrealistically large spatial scale, and that this in fact worsens the simulation.

\section{Conclusions}

A regional atmosphere model has been used over a large North Atlantic domain to investigate the sensitivity of the storm track to the spatial and temporal resolution of SST data. Firstly, it is clear that the model reproduces the general structure of the storm track very well when compared to the ERA-40 data, which are used as lateral boundary conditions.

This work has shown that, in general, the storm track is sensitive to the SST data used as a lower boundary condition. Large-scale differences between the low-resolution R02 and high-resolution R07 data motivated the use of separate experiments to assess the impact of improving spatial and temporal resolution within the same dataset. These show that the storm track is sensitive to both the temporal and spatial resolution of the SSTs. Increasing the horizontal resolution acts to shift the storm track partly off the North American coast and onto the Gulf Stream temperature gradients. Increasing the temporal resolution from monthly to weekly (or daily) increases the storm activity over the Gulf Stream and leads to significant changes in activity over Europe. The character of these European changes is similar in experiments with both SST datasets, but the location which is affected most does depend on the dataset used.

This work has shown that the storm track is sensitive to changes in the spatial and temporal resolution of SSTs, though interestingly an improvement in resolution, particularly temporal, does not always lead to an improved simulation when compared to observations. Increasing the temporal resolution of SST data of low spatial resolution can lead to fast SST variations of unrealistically large length scales.

The storm track sensitivity shown here is small, but significant, and while the changes are generally in the detailed structure of the storm track, some of these changes occur over populated regions, and they may be important. For example, since the response of the North Atlantic storm track to increased greenhouse gas forcing is often subtle (Bengtsson et al. 2006), it may be important that the detailed structure of the storm track is represented as well as possible in climate models. Also, since the effect of increased spatial resolution is to pull the start of the storm track off the North American coast and onto the strong SST gradient, this could mean that the storm track is more sensitive to SST variability than is apparent in low-resolution models.

It should also be remembered that, since the lateral boundary conditions constrain roughly $50 \%$ of the largescale variability in the model, the results shown here may be an underestimate of the impact of increasing SST resolution. In addition, Chelton et al. (2006) found that fine-scale wind structure in the atmospheric boundary layer is underestimated even in high resolution models driven by high resolution SSTs, and Song et al. (2009) have shown that this arises due to an underestimate of vertical diffusion in the boundary layer. Although these studies used different models to that used here, it is possible that limitations in the boundary layer scheme could also be leading to an underestimate of the sensitivity of the storm track to SST resolution in our experiments.

Acknowledgments This work was performed as part of the NERC RAPID Climate Change programme and was supported by the Defra and MoD Integrated Climate Programme-(Defra) GA01101, (MoD) CBC/2B/0417_Annex C5. We would like to thank Richard Reynolds for help and advice on the use of the SST data, and Dudley Chelton and the anonymous reviewers for comments which have helped to improve the paper.

\section{References}

Bengtsson L, Hodges KI, Roeckner E (2006) Storm tracks and climate change. J Clim 19:3518-+. doi:10.1175/JCLI3815.1

Brayshaw DJ, Hoskins B, Blackburn M (2008) The storm track response to idealised SST perturbations in an aquaplanet GCM. J Atmos Sci 65:2842-2860

Chelton DB, Wentz FJ (2005) Global microwave satellite observations of sea surface temperature for numerical weather prediction and climate research. Bull Am Meteorol Soc 86:1097-1115

Chelton DB, Freilich MH, Sienkiewicz JM, von Ahn JM (2006) On the use of QuikSCAT scatterometer measurements of surface winds for marine weather prediction. Mon Weather Rev 134:2055-+. doi:10.1175/MWR3179.1

Denis B, Cote J, Laprise R (2002) Spectral decomposition of twodimensional atmospheric fields on limited-area domains using the discrete cosine transform (DCT). Mon Weather Rev 130:1812-1829

Doyle JD, Warner TT (1993) The impact of sea surface temperature resolution on mesoscale coastal processes during GALE IOP 2. Mon Weather Rev 121:313-334

Hodges KI (2008) Confidence intervals and significance tests for spherical data derived from feature tracking. Mon Weather Rev 136:1758-1777

Hoskins BJ, Hodges KI (2002) New perspectives on the northern hemisphere winter storm tracks. J Atmos Sci 59:1041-1061

Hoskins BJ, Valdes PJ (1990) On the existence of storm-tracks. J Atmos Sci 47:1854-1864

Hoskins BJ, James IN, White GH (1983) The shape, propagation and mean-flow interaction of large-scale weather systems. J Atmos Sci 40:1595-1612

Inatsu M, Mukougawa H, Xie SP (2002) Tropical and extratropical SST effects on the midlatitude storm track. J Meteorol Soc Jpn 80(4B):1069-1076 
Jones RG, Murphy JM, Noguer M (1995) Simulation of climate change over Europe using a nested regional-climate model. I: assessment of control climate, including sensitivity to location of lateral boundaries. Q J R Meteorol Soc 121:1413-1449

Jones RG, Noguer M, Hassell DC, Hudson D, Wilson SS, Jenkins GJ, Mitchell JFB (2004) Generating high resolution climate change scenarios using PRECIS. Met Office Hadley Centre, Exeter

Kuo YH, Low-Nam S, Reed RJ (1991) Effects of surface energy fluxes during the early development and rapid intensification stages of seven explosive cyclones in the Western Atlantic. Mon Weather Rev 119:457-475. doi:10.1175/1520-0493(1991)119

Minobe S, Kuwano-Yoshida A, Komori N, Xie SP, Small RJ (2008) Influence of the Gulf Stream on the troposphere. Nature 452:206-209. doi:10.1038/nature06690

Nakamura H, Sampe T, Tanimoto Y, Shimpo A (2004) Observed associations among storm tracks, jet streams and midlatitude oceanic fronts. In: Wang C, Xie SP, Carton JA (eds) Earth's climate: the ocean-atmosphere interaction, vol 147. AGU Geophysical Monograph Series

Nakamura H, Sampe T, Goto A, Ohfuchi W, Xie SP (2008) On the importance of mid-latitude oceanic frontal zones for the mean state and dominant variability in the tropospheric circulation. Geophys Res Lett 35. doi:10.1029/2008GL034,010

Reynolds RW, Rayner NA, Smith TM, Stokes DC, Wang W (2002) An improved in situ and satellite SST analysis for climate. J Clim 15:1609-1625

Reynolds RW, Liu C, Smith TM, Chelton DB, Schlax MG, Kasey KS (2007) Daily high-resolution blended analyses for sea surface temperature. J Clim 20:5473-5496

Sardeshmukh PD, Hoskins BJ (1984) Spatial smoothing on the sphere. Mon Weather Rev 112:2524-2529

Semmler T, Varghese S, McGrath R, Nolan P, Wang S, Lynch P, O'Dowd C (2008) Regional model simulation of North Atlantic cyclones: present climate and idealised response to increased sea surface temperature. J Geophys Res 113. doi:10.1029/2 006JD008213
Simmons AJ, Hoskins BJ (1978) The life cycles of some nonlinear baroclinic waves. J Atmos Sci 35:414-432

Song Q, Chelton DB, Esbensen SK, Thum N, O’Neill LW (2009) Coupling between sea surface temperature and low-level winds in mesoscale numerical models. J Clim 22:146-164

Taguchi B, Nakamura H, Nonaka M, Xie SP (2009) Influences of the Kuroshio/Oyashio extensions on air-sea heat exchanges and storm track activity as revealed in regional atmospheric model simulations for the 2003/4 cold season. J Clim (submitted)

Taylor KE, Williamson D, Zwiers F (2000) The sea surface temperature and sea-ice concentration boundary conditions for AMIP II simulations. PCMDI Report No 60

Thiébaux J, Rogers E, Wang W, Katz B (2003) A new high-resolution blended real-time global sea surface temperature analysis. Bull Am Meteorol Soc 84:645-656

Uppala SM et al (2005) The ERA-40 re-analysis. Q J R Meteorol Soc 131(612):2961-3012

Warner TT, Lakhtakia MN, Doyle JD (1990) Marine atmospheric boundary layer circulations forced by Gulf Stream sea surface temperature gradients. Mon Weather Rev 118:309-323

Wentz FJ, Gentemann C, Smith D, Chelton D (2000) Satellite measurements of sea surface temperature through clouds. Science 288:847-850

Xie SP (2004) Satellite observations of cool ocean atmosphere interaction. Bull Am Meteorol Soc 85(2):195-208

Xie SP, Hafner J, Tanimoto Y, Liu WT, Tokinaga H, Xu H (2002) Bathymetric effect on the winter sea surface temperature and climate of the yellow and east China seas. Geophys Res Lett 29:81-1

Yamamoto M, Hirose N (2007) Impact of SST reanalyzed using OGCM on weather simulation: a case of a developing cyclone in the Japan Sea area. Geophys Res Lett 34:5808-+. doi:10.1029/ 2006GL028386

Zwiers FW, von Storch H (1995) Taking serial correlation into account in tests of the mean. J Clim 8:336-351 